\title{
Influence of surface treatments on CdSe thin films in photoelectro- chemical solar energy conversion
}

\author{
SHIKHA MISHRA, SANJAY TIWARI* and B P CHANDRA \\ Department of Post Graduate Studies and Research in Physics, R.D. University, Jabalpur \\ 482001, India \\ *Department of Physics, Govt. Autonomous Science College, Jabalpur 482001, India \\ MS received 7 February 1994; revised 8 April 1994
}

\begin{abstract}
Photovoltaic performance "of semiconductor-liquid junction solar cell using electro-codeposited thin film $n$-CdSe is found to improve significantly by proper surface treatments. The solid state parameters of annealed films are calculated and compared with those of unannealed film-based cells. Chemical etching is found to improve short circuit current and fill factor whereas photoelectrochemical etching technique improves the stability of photoanode in polysulphide electrolyte. Annealing promotes incipient fusion of small crystallites, thus reducing the grain boundaries which are known to act as recombination centres for minority carriers and trapping centre for majority carriers. The conversion efficiency and stability are found to improve by chemical etching of the semiconductor layer because chemical etching pins Fermi level of CdSe photoanode and promotes exchange current density.
\end{abstract}

Keywords. PEC solar cells; CdSe; annealing; photoelectrochemical (photo) etching; cell parámeters.

\section{Introduction}

Photoelectrochemical solar cells based on $n$-type cadmium chalcogenides in polysulphide redox electrolytes have attracted much attention as practical solar energy, convertors. The key findings are that polysulphide ions enable these semiconductor materials to be photoelectrochemically stable and make the flat band potential of these electrodes shift towards the cathodic direction. Polycrystalline cadmium selenide has shown great promise as a photoelectrode for solar energy conversion through PEC cells. However, because of inherent defects present in polycrystalline materials, a considerable portion of the photogenerated charge carriers is lost due to recombination particularly in the intergrain region of the bulk. This results in overall poor performance of the cell. Research efforts to avoid grain boundary effects have been made by several workers by subjecting the films to chemical treatment (Ramprakash et al 1984; Yoneyama et al 1985). Bandyopadhyay and Chaudhuri (1985) and Pandey et al (1987) have shown that annealing of thin film cadmium chalcogenide photoanode leads to a substantial increase in the short circuit current and open circuit voltage and hence overall conversion efficiency.

In this case, due to surface affinity and formation of a thin layer of sulphur, a few layers of CdS are formed on CdSe which give rise to dislocation or grain boundaries near the surface which act as recombination centres and decrease the efficiency. Heller et al (1978) have suggested that this ion exchange can be suppressed by adding

\footnotetext{
*For correspondence
} 
elemental selenium to the redox electrolyte. Here we report our observations on the influence of annealing, chemical etching and photochemical etching treatments on photovoltaic performance of thin films of cadium selenide.

\section{Experimental}

The cadium selenide films were cathodically deposited on titanium substrates at $30^{\circ} \mathrm{C}$ under galvanostatic conditions in an aqueous acidic electrolyte consisting of AR grade $0.2 \mathrm{M} \mathrm{CdSO}_{4}$ and $6 \times 10^{-3} \mathrm{M} \mathrm{SeO}_{2}$. The films were deposited at $7 \mathrm{~mA} / \mathrm{cm}^{2}$ current density. Film thicknesses were $\sim 3$ to $4.5 \mu \mathrm{m}$. Annealing was carried out in air at $370^{\circ} \mathrm{C}$. Annealed films were then etched chemically and photoelectrochemically in $\mathrm{HCl}: \mathrm{HNO}_{3}: \mathrm{H}_{2} \mathrm{O}:: 3: 1: 12$ (by volume) using graphite as counterelectrode and then thoroughly rinsed with distilled water.

Measurements of optoelectronic properties were done using a liquid rectifying contact obtained by dipping $n$-CdSe films in an electrolyte consisting of $\mathrm{NaOH}, \mathrm{Na}_{2} \mathrm{~S}$ and $S$ (each $1 \mathrm{M}$ ). A conventional three-electrode configuration was used with graphite as a counterelectrode and saturated calomel electrode as reference electrode. The cell had a flat window for illumination of the film. Before dipping, copper leads were soldered on the back surface of the titanium substrates and a common epoxy was applied to seal the back and the sides. The current-voltage characteristics were studied in dark as well as under illumination using a $200 \mathrm{~W}$ tungsten lamp.

\section{Results}

From thermoelectric power measurements, all the CdSe films were found to be $n$-type. Figure 1 shows the $I-V$ characteristics for unannealed, annealed and chemically etched CdSe films. The corresponding solar cell parameters are recorded in table 1. It is clear from table 1 that values of all the solar cell parameters increase on annealing the films, while with chemical etching values of $I_{\mathrm{SC}}$ and fill factor increase further but $V_{\mathrm{OC}}$ decreases slightly. It is also found that stability of the photoanode increases significantly after photoelectrochemical etching.

Figure 2 shows the plot of photocurrent efficiency vs voltage for annealed and unannealed CdSe films. From the photocurrent onset point we estimated the flat band potential for unannealed and annealed films to be -1.39 and $-1.2 \mathrm{~V}$ (vs SCE), respectively. Taking $E_{f, \text { redox }}$ for the sulphide/polysulphide couple as $-0.71 \mathrm{~V}$, band bendings were calculated. Plots of $\ln (1-\phi)$ vs $\left(E_{f, \text { redox }}-E_{f b}\right)^{1 / 2}$ are shown in figure 3. From these plots values of donor concentration $N_{D}$ and hole diffusion length $L_{P}$ are calculated from the slope and intercept respectively using a value for absorption coefficient of $3 \times 10^{4} \mathrm{~cm}^{-1}$ (Bandyopadhyay and Chaudhuri 1985). These values of donor concentration, hole diffusion length and band bending are recorded in table 2 .

Comparison of stabilities of chemically etched and photochemically etched CdSe films is shown in figure 4. It is seen that the photoetched electrode is more stable than the chemically etched one.

Figure 5 shows that photoetching modifies the photocurrent vs light intensity behaviour. It is seen that under conditions where the short circuit current $I_{\mathrm{SC}}$ of chemically etched electrode showed saturation with the light intensity $I_{\text {in }}$, a linear dependence of $I_{\mathrm{SC}}$ vs $I_{\text {in }}$ is observed with the photoetched electrode. 


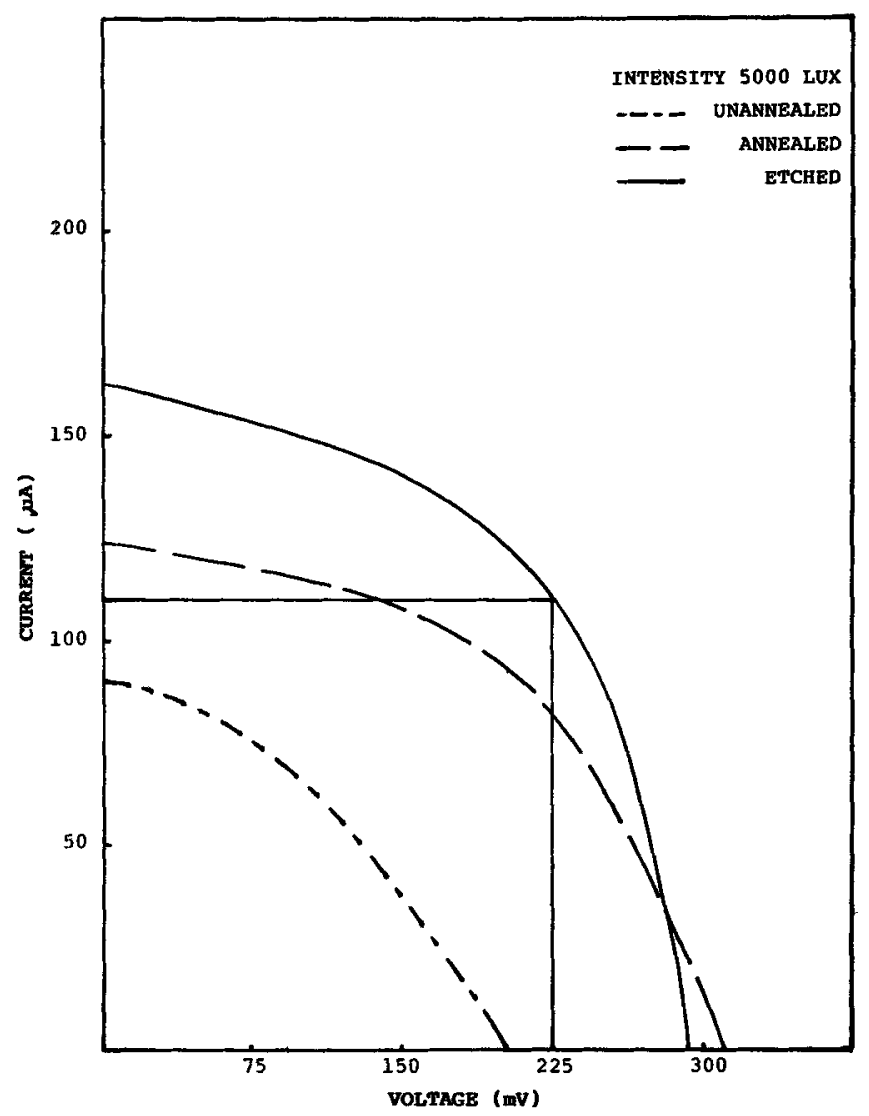

Figure 1. I-V characteristics of CdSe-based PEC solar cells for unannealed, annealed and etched films.

Table 1. Solar cell parameters using unannealed, annealed and annealed and etched CdSe films.

\begin{tabular}{lcrrc}
\hline Nature of films & $\begin{array}{c}V_{\text {oc }} \\
(\mathrm{mV})\end{array}$ & $\begin{array}{c}I_{\text {sc }} \\
(\mu \mathrm{A})\end{array}$ & FF & $\begin{array}{c}\eta \text { Efficiency } \\
(\%)\end{array}$ \\
\hline Unannealed & 200 & 90 & 0.33 & 0.84 \\
Annealed & 312 & 118 & 0.50 & 2.58 \\
Annealed and chemically etched & 290 & 163 & 0.53 & 3.45 \\
\hline
\end{tabular}

\section{Discussion}

Improvement in photoelectrochemical performance of solar cells on annealing CdSe films has been reported by several workers (Cardona et al 1967; Butler 1977; Sprunken et al 1980; Gautron and Lemasson 1982). The effect of annealing can be explained by using Gartner's (1959) model, in which recombination in the space charge region as well as interface kinetics were ignored. The expression for photocurrent can be written 

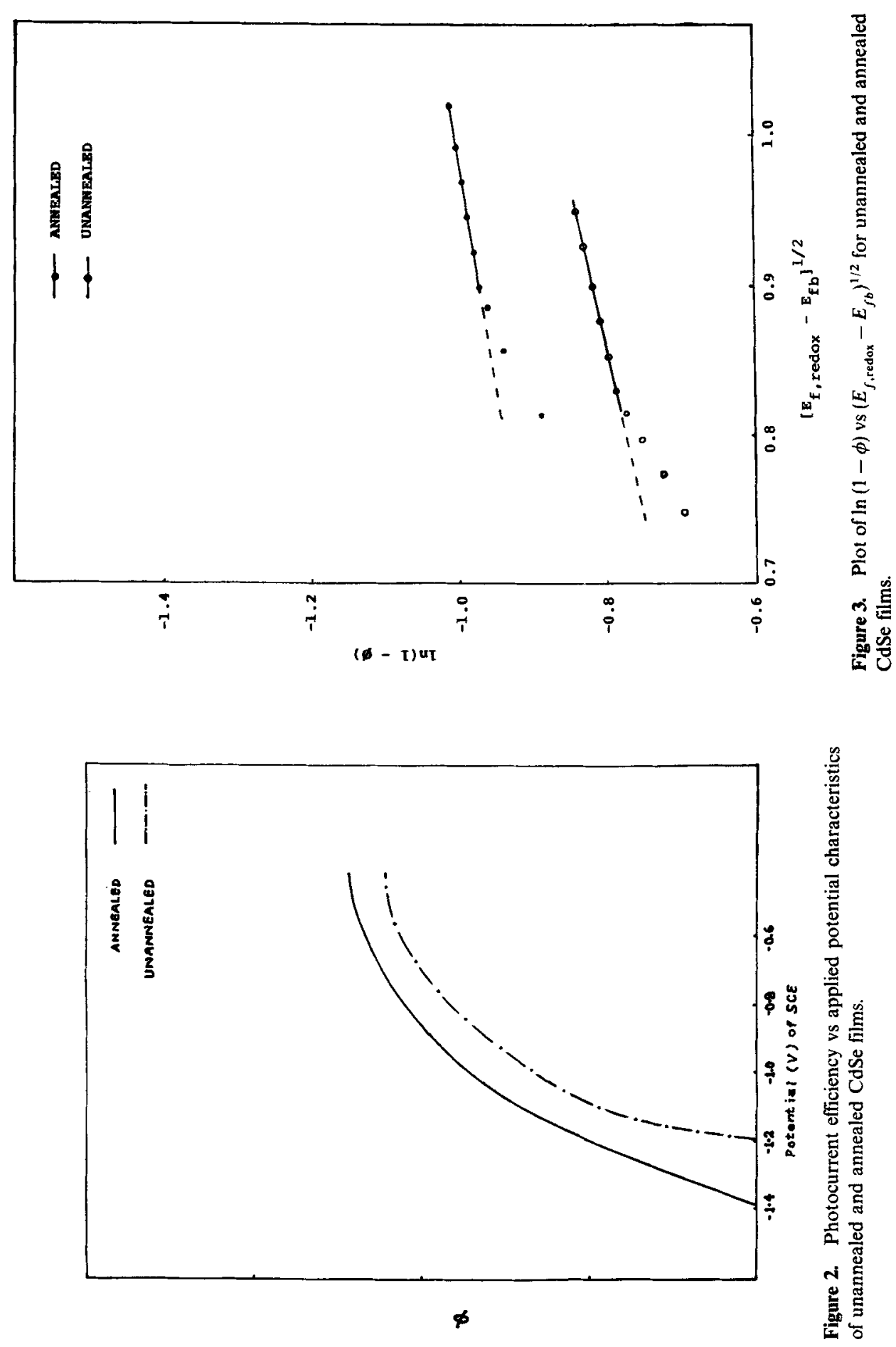
Table 2. Solid state parameters of annealed and unannealed CdSe films.

\begin{tabular}{lccc}
\hline Nature of films & $V_{b}$ & $N_{D}\left(10^{13} \mathrm{~cm}^{-3}\right)$ & $L_{P}\left(10^{-5} \mathrm{~cm}\right)$ \\
\hline Unannealed & $0.49 \mathrm{~V}$ & 12.6 & 0.008 \\
Annealed & $0.68 \mathrm{~V}$ & 13.2 & 0.015 \\
\hline
\end{tabular}

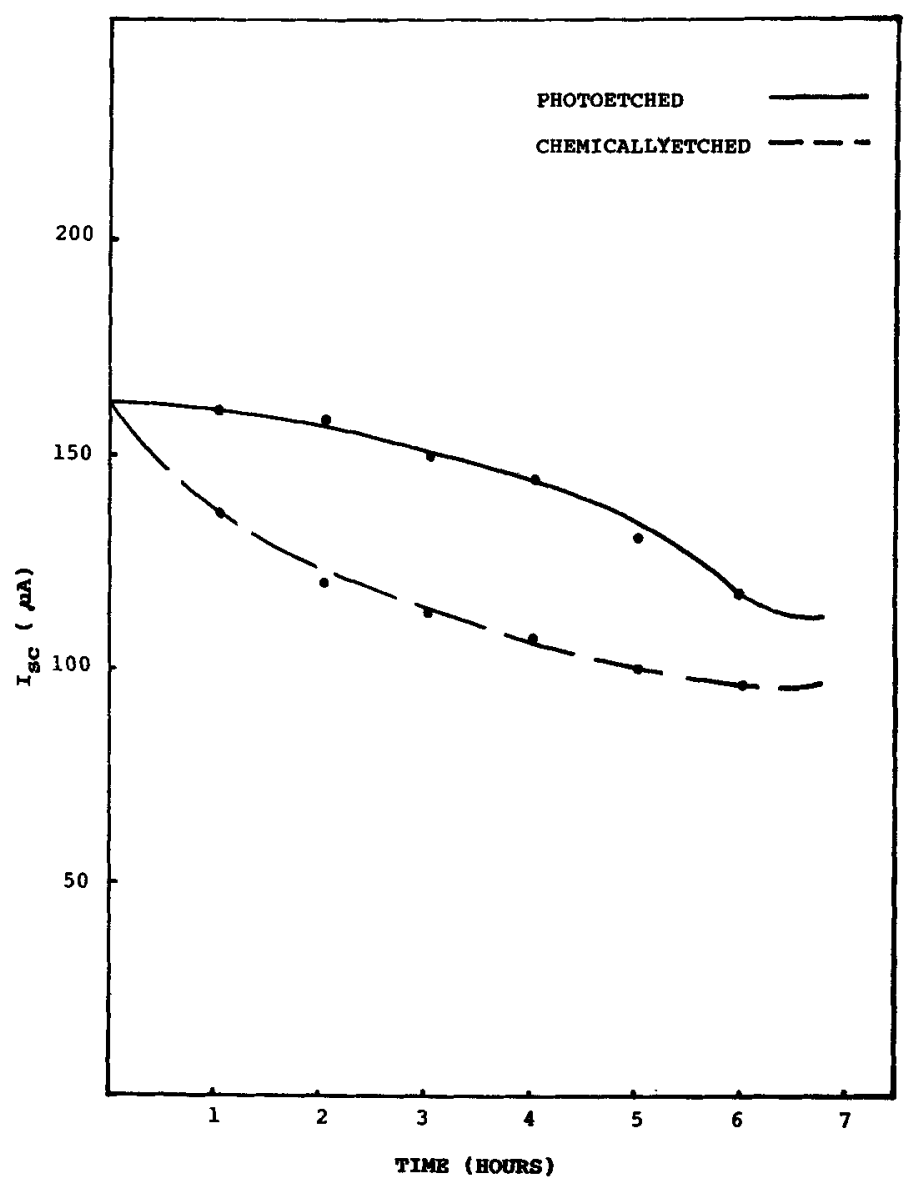

Figure 4. Comparison of the stabilities of chemically etched and photoetched CdSe-based PEC solar cells.

as

$$
J_{\mathrm{ph}}=e \phi_{0}\left(1-\frac{\exp (-\alpha W)}{1+\alpha L_{\mathrm{P}}}\right)
$$

where $\phi_{0}$ is the incident photoflux, $\alpha$ the absorption coefficient, and $L_{P}$ the minority carrier diffusion length. The depletion layer width $W$ is given by

$$
W=\left(\frac{2 \varepsilon \varepsilon_{0} \psi_{S}}{e N_{D}}\right)^{1 / 2}
$$




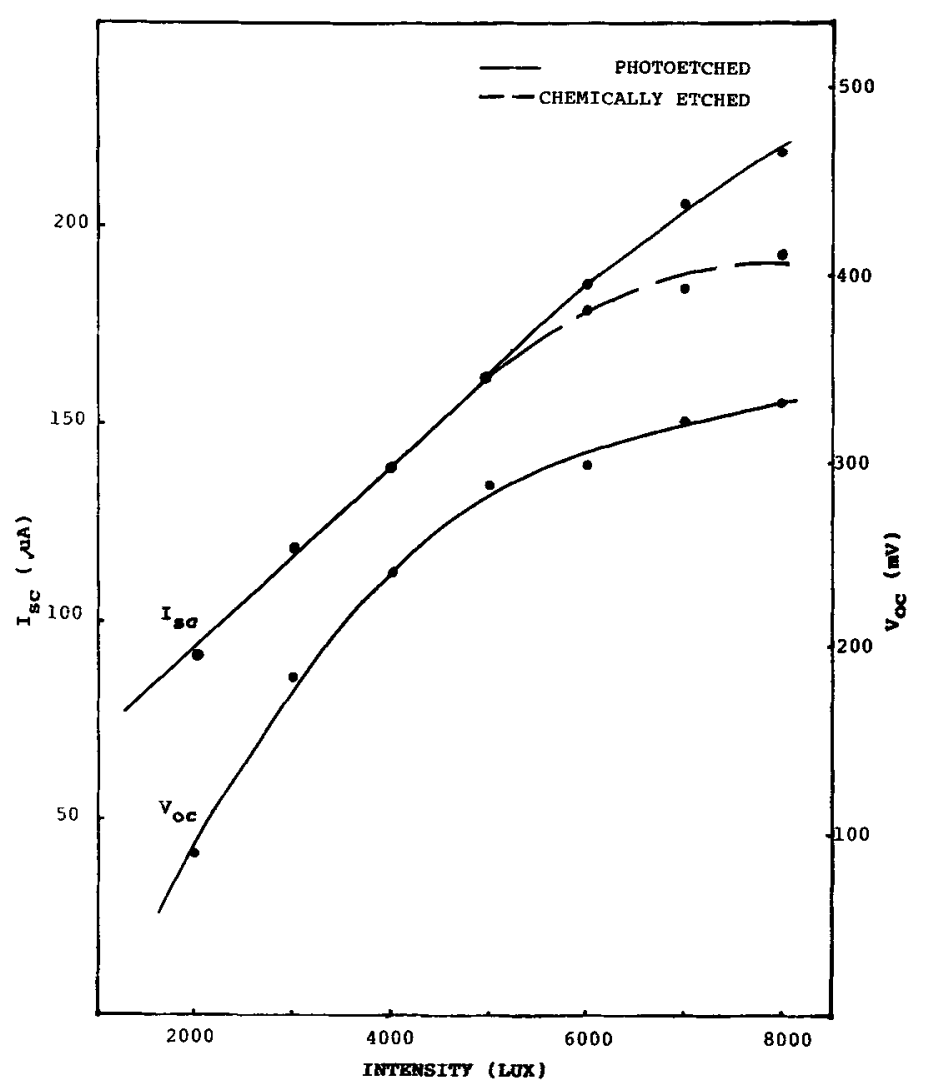

Figure 5. Effect of incident light intensity on $V_{\alpha c}$ and $I_{S C}$ of CdSe-based PEC solar cells.

where $\varepsilon_{0}$ is the permittivity in vacuum, $\varepsilon$ the dielectric constant of semiconductor, $e$ the electronic charge, $N_{D}$ the donor density, and $\psi_{S}$ the voltage drop across the space charge layer given by $E_{f, \text { redox }}-E_{f b}$.

The flat band potential $\left(E_{f b}\right)$ gives the measure of the maximum open circuit voltage $V_{\text {oc }}$ which can be obtained. The flat band potential is determined from the photocurrent onset (figure 2). Taking $E_{f, \text { redox }}$ for the sulphide/polysulphide couple as $-0.71 \mathrm{~V}$, the band bending comes out to be 0.68 and $0.49 \mathrm{~V}$ for annealed and unannealed film respectively. It is clear from the above values of band bending for annealed and unannealed films that annealing results in larger band bending hence higher $V_{\text {oC }}$ values.

The expression for photocurrent efficiency can be obtained from (1) as

$$
\phi=1-\exp \left[1-\frac{\exp (-\alpha W)}{1+\alpha L_{P}}\right]
$$

From (2) and (3),

$$
\ln (1-\phi)=-\alpha\left(\frac{2 \varepsilon \varepsilon_{0} \psi_{S}}{e N_{D}}\right)^{1 / 2}-\ln \left(1+\alpha L_{P}\right) .
$$


It is clear from table 2 that $L_{P}$ and $N_{D}$ increase on annealing CdSe films. The increase in $N_{D}$ and $L_{P}$ due to heat treatment of the films causes enhancement in $I_{S C}$. Due to the increase in $I_{\mathrm{SC}}$ and $V_{\mathrm{OO}}$ of annealed films overall conversion efficiency also increases. The diffusion length is a function of lifetime and mobility, which is related to recombination and trapping within the grains as well as the grain boundaries. The grain boundaries are known to act as recombination centres for minority carriers and trapping centre for majority carriers. Because the annealing promotes incipient fusion of smaller crystallites, thus reducing the grain boundaries, the diffusion length will also increase. In case of CdSe, it has been observed that annealing also causes removal of excess Se leading to a reduction in $\mathrm{Se} / \mathrm{Cd}$ ratio at the surface. Thus, the improvement in cell performance through increase in depletion layer width under the influence of annealing is attributed to the removal of recombination centres in the form of Se acceptors.

Etching acts as deliberate corrosion as it removes selectivity, surface states and dislocations (Paranthaman et al 1980), thus favouring large improvements in PEC behaviour of the semiconductor electrodes. Tenne and Muller (1983) have found that etching of cadmium chalcogenide electrodes improves their efficiency and stability in polysulphide electrolyte.

Due to removal of surface states by etching, the Fermi level of the semiconductor is pinned at a certain value relative to the edge of the conduction or valence band, so that the changes in $E_{f \text {,redox }}$ of the electrolyte do not alter the observed photovoltage. In case of semiconductor electrolyte interface, if there is no pinning, i.e. there is ideal behaviour, then $E_{f b}$ (flat band potential) is constant. It is known that the built-in potential $V_{b}$ is related to the $E_{f, \text { redox }}$ and is given by the relation

$$
V_{b}=E_{f, \text { redox }}-E_{f b} \text {. }
$$

This equation shows that $V_{b} \propto E_{f, \text { redox }}$, for the ideal case, i.e. when there is no pinning of fermi level. However, in presence of Fermi level pinning, as $E_{f \text {,redox }}$ varies, opposite results are obtained. So, when $E_{f, \text { redox }} \propto E_{f b}, V_{b}$ will remain constant and thereby the photovoltage will remain constant.

With $n$-CdSe, pinning occurs after an oxidizing etch which removes excess of $\mathrm{Cd}$ free metal which acts as a recombination centre and lowers the photocurrent.

In CdSe crystals the main effect of photoetching is due to the formation of pits which increase the area of the junction by about $50 \%$. This increase in the area reduces the reflectivity of the surfaces approximately by $10 \%$ and improves the rate of the hole-electrolyte reaction considerably. Photoetching, which increases the surface roughness of the electrode, has the effect of reducing the effective surface concentration of the hole per unit area of the junction. This ensures an adequate supply of adsorbed sulphide ions which results in an improved stability.

Roughness $S^{\prime}$ is the ratio between the real surface area of the junction and its geometrical surface area and can be expressed as

$$
S^{\prime}=\int_{0}^{L} S_{P} \mathrm{~d} x,
$$

where $\mathrm{d} x$ is the distance from the surface and $S_{P}$ the specific surface area of a unit volume of the electrode close to the electrode surface. By increasing the roughness density of sites by $S^{\prime}\left(S^{\prime}>1\right)$, the relative surface concentration of reduced species $\left(\theta_{\text {red }}\right)$ 
increases from $\theta_{\text {red }}$ to $S^{\prime} \theta_{\text {red }}$. Using Tenne et al's (1983) model, the expression for the excess concentration of holes on the surface $\left(P_{s}\right)^{*}$, can be written as

$$
O=\frac{\mathrm{d} P_{s}^{*}}{\mathrm{~d} t}-J_{\phi}-S_{r} P_{s}^{*}-K_{1} P_{s}^{*}-K_{2} P_{s}^{*} \theta_{\mathrm{red}} S
$$

where $J_{\phi}$ is the hole flux arriving at the semiconductor surface and is equal to $J_{\text {reg }}+J_{\text {cor }}+J_{\text {rec }}$, where $J_{\text {reg }}$ is regenerative photocurrent, $J_{\text {cor }}$ the corrosion current and $J_{\text {rec }}$ the recombination current; $S_{r}$ is the surface recombination velocity; and $K_{1}$ and $K_{2}$ are the constants for the photocorrosion reactions and for the regenerative oxidation of the adsorbed electrolyte.

From (7), we get

$$
P_{s}^{*}=\frac{J_{\phi}}{S_{r}+K_{1}+K_{2} \theta_{\text {red }} S} .
$$

Hence the regenerative current may be written as

$$
J_{\text {reg }}=K_{2} P_{s}^{*} \theta_{\text {red }} S^{\prime}=\frac{S^{\prime} K_{2} J_{\phi} \theta_{\text {red }}}{\left(S_{r}+K_{1}+K_{2} \theta_{\text {red }} S^{\prime}\right)} .
$$

It is seen that $J_{\text {reg }}$ increases with increase in $S^{\prime}$ while recombination current and corrosion current decrease with $S^{\prime}$. Thus stability increases with photoetching.

Saturation of the short-circuit current vs light intensity of chemically etched CdSe photoanode proved to be a good indication for slow kinetics of the hole-electrolyte reaction leading to photocorrosion. Photoetching modifies photocurrent vs light intensity behaviour. The increase in incident light intensity beyond saturation level of $I_{\mathrm{SC}}$ for chemically etched CdSe photoanode results in photocorrosion of photoanode while photoetched electrodes are found stable at these high intensities, because photoetching has an effect of reducing the effective surface concentration of the holes per unit area of the junction. This ensures an adequate supply of adsorbed sulphide ions which results in improved stability. By increasing the light intensity, the sulphide ions from the interface are depleted, therefore the holes have no redox electrolyte to oxidize and they are impeded on the electrode surface, so their probability to oxidize the crystal increases.

\section{Conclusion}

Annealing of CdSe films shifts the flat band potential from $-1 \cdot 2$ to $-1 \cdot 39$. A higher open-circuit voltage will therefore result on annealing. The overall conversion efficiency is more sensitive to minority carrier (hole) diffusion length which also increases as a result of annealing as well as chemical etching. The increase in hole diffusion length on annealing has been attributed to increased grain size while chemical etching removes excess of Cd metal acting as recombination centre. As a result of chemical etching Fermi level pinning of photoanode occurs, thereby photovoltage also remains constant. Photoelectrochemical etching has an effect of reducing the effective surface concentration of the holes per unit area of the junction which ensures adequate supply of adsorbed sulphide ions which results in improved stability. 


\section{References}

Bandyopadhyay T K and Chaudhuri S R 1985 Phys. Status Solidi (a) 92637

Butler M A 1977 J. Appl. Phys. 481914

Cardona M, Shaklee K L and Pollak F H 1967 Phys. Rev. 154696

Gartner W W 1959 Phys. Rev. 11684

Gautron J and Lemasson P 1982 J. Cryst. Growth 59332

Heller A, Chang K C and Miller B 1978a J. Am. Chem. Soc. 100684

Heller A, Chang K C and Miller B 1978b J. Electrochem. Soc. 1251156

Pandey R K, Rooz A J N and Gore R B 1987 J. Phys. D. : Appl. Phys. 201059

Paranthaman M, Aravamudan G and Subba Rao G K 1980 Indian J. Technol. 24399

Ramprakash Y, Bose D N and Basu S 1984 Surf. Sci. 145175

Sprunken H R, Schumacher R and Schindler R N 1980 Disc. Faraday Soc. 7055

Tenne R and Muller N 1983 J. Electrochem. Soc. 852

Tenne R, Muller N, Mirousky Y and Lando D 1983 J. Electrochem. 130852

Yoneyama H, Azuma H and Tamura H J 1985 J. Electrochem. Soc. 186247 